\title{
MIPT JACKET (Mentally Illed Patient Tracking)
}

\author{
Navita Agarwal ${ }^{* 1}$, Shubham Bhatt ${ }^{\# 2}$, Shubham Gupta ${ }^{\# 3}$, Rishabh Agarwal ${ }^{\# 4}$, Mohd. Anzar ${ }^{\# 5}$ \\ *1 Assistant Professor, Department of Computer Science \& Engineering, MIT, Moradabad, U.P, India \\ \#2,3,4,5 Student, Department of Computer Science \& Engineering, MIT, Moradabad, U.P, India
}

\begin{abstract}
In health care, IOT as a key player that furnishes better healthcare facilities to the affected, doctors and hospitals as well at anytime and anywhere. The proposed system consists of various medical devices such as sensors and mobile based application which communicates via network connected devices. These devices will monitor and record patients' health data and various medical information. Different types of sensors are used like Temperature Sensor, Seizure Sensor, GPS Sensor, GSM Sensor and Pulse Sensor. The proposed paper will provide instant information about the patient's current health status and full medical information in case any medical emergency arises both to the family members and the hospital. The collected information will predict patient's chronic disorders or other diseases such as heart attacks in preliminary stage itself using the data mining techniques. MIPT Jacket technology provides greater visibility into every patient's safe zone and improves patient's safety by identifying the gaps in internal hospital processes and recovering them. Instant medical help will be provided to patient in non - safe zone also via sensors.
\end{abstract}

Keywords - Internet of Things (IoT), IoT in Healthcare, Patient Monitoring, Raspberry Pi, Smart Health Monitoring.

\section{INTRODUCTION}

The Internet of things (IoT) is the inter-connection of devices, applications, sensors and network connectivity that enhances these entities to gather and exchange data. The different characteristics of Internet of Things in the healthcare system are the constant monitoring processes a patient through checking

various parameters and also infer best results from the history of such constant monitoring. Many such devices equipped with medical sensors are present in the ICUs now-a-days. There could be cases where the doctor couldn't be alarmed in time when there is an exigency, despite of 24 hours of monitoring.

There might be hurdles in sharing the data and information with the specialist doctors and the concerned family members and relatives. The technology that enhances these features is already available but is not accessible and affordable by most of the people in developing countries such as India. Therefore, solutions of problems can be extension to the current devices which don't have these facilities.

This paper describes a MIPT Jacket [1] controlled by RPi. RPi is a small payment card-sized single-board microcontroller made to enhance the basic computer science education in colleges and developing nations. A system is designed to continuously monitor the distinguished parameters such as heart rate, blood pressure and body temperature. The information is stored on a cloud server database and can be displayed through an online website or mobile application by authorized personnel only. This idea is an absolute and cheap method for the system using Raspberry pi. The proposed system can update the data online and send an alert to the doctors for any abnormality and also predict if the patient is having any disease. The proposed system has great future scope as the data collected by monitoring is so valuable and can be used for any kind of research by the medical community. 


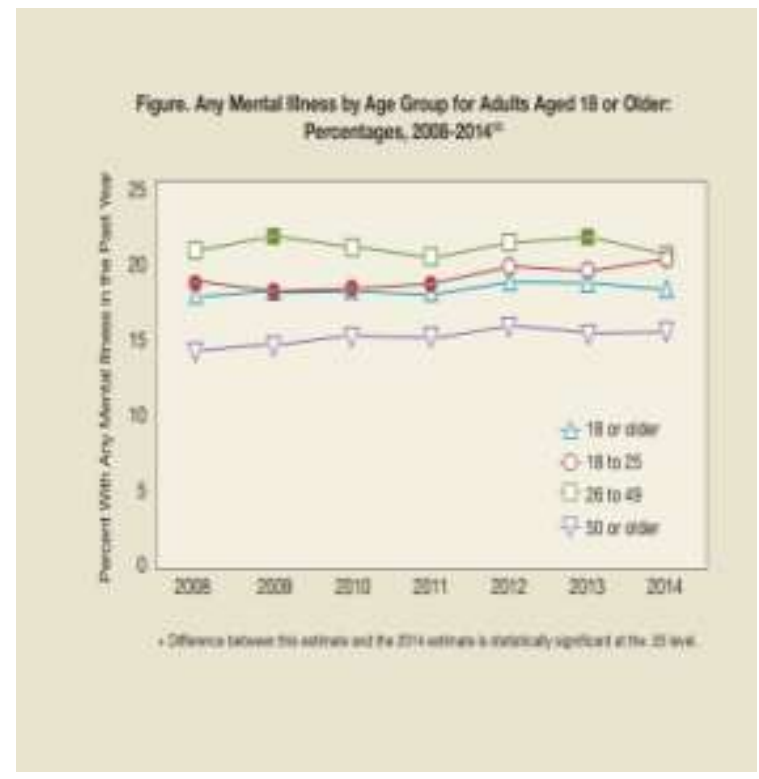

Fig. 1.1 Representation of some mental illness

The major aim of the proposed system can be summarized as following:

- To retrieve the real-time medical information about a patient via IoT.

- $\quad$ Processing and classification of information gathered about the patient.

- $\quad$ To construe and anticipate any disease or disorder in preliminary stage itself using the data mining techniques that will also provide the approach advantageous for decision making.

- $\quad$ To provide IoT based health care resoslutions at anytime and anywhere.

\section{LITERATURE REVIEW}

A number of researchers have proposed various models for IoT in Healthcare and the prediction of various types of diseases using various techniques. Ahn et al. [2] implemented a system for measuring the physiological signals in sitting position such as ECG and BCG by using a smart chair that senses the non- constrained biosignals and can be monitored using a monitoring system.

Almotiri et al. [3] proposed a system of $m$-health that uses mobile devices to collect real-time data from patients in and store it on network servers connected to internet enabling access only to a certain specific clients. This data can be used for the medical diagnosis of patients and is achieved by using a number of wearable devices.

Barger et al. [4] made a smart house facility using a sensor network to monitor and track the movements of the patient in hoke and a prototype of the same is also being tested. The primary objective of their work is to check if their system is capable to outsmart the behavioral patterns.

Chiuchisan et al. [5] proposed a framework to prevent the threats to patient in smart ICUs. The proposed system intimates the patient's relatives and doctors about any inconsistency in their health status or their body movements and also about the atmosphere of the room so that the necessary precautionary measures can be taken.

Dwivedi et al. [6] developed a framework in order to secure the clinical information that has to be transmitted over the internet for Electronic Patient Record (EPR) systems in which they propose a multi-layered healthcare 
information system framework which is a combination of Public Key Infrastructure, Smartcard approach and Biometrics storage technologies.

\section{PROPOSED SYSTEM}

In this paper, an automatic system is proposed that will monitor patient's heart rate, body temperature, and blood pressure and body movements. Then the existing system will be extended in order to predict if the patient has been suffering from any chronic disease or disorder using several health parametric quantities and also several other symptoms that would be obtained by the system.

In this paper, a MIPT (Mentally Illed Person Tracking) Jacket using Raspberry Pi with GPS sensors installed andGSM or CDMA connectivity is proposed. This device will help various families or organizations to keep an eye on the mentally ill person. For this purpose, a range for the patients to walk around can be involved.

\section{GPS}

\section{HARDWARE PROPOSED TO BE USED}

Movement Monitor will observe abnormal and faster movements that may occur during the night, and this will sound a high-frequency alarm if these causes continue past a preset amount of time. The Movement Monitor consists of a film-like sensor and a control unit.

\section{GSM}

GSM is global system for mobile communication (GSM). GSM is exposed and digital cellular tech used for broadcasting mobile voice and data serves engages at the $850 \mathrm{MHz}, 900 \mathrm{MHz}, 1800 \mathrm{MHz}$ and $1900 \mathrm{MHz}$ frequency bands.

\section{Temperature Sensor}

Temperature sensor is a gimmick, to measure the temperature through an electrical signal, it requires a thermocouple junction or RTD (Resistance Temperature Detectors). If the deviation in voltage is amplified, the analog signal is generated by the device and it is directly relative to the temperature.

\section{Pulse Sensor}

Motion Monitor is designed to detect faster unnatural motions that may occur during the night, and will sound a high-frequency alarm if these motions continue past a predetermined amount of time. The Emfit MM, Motion Monitor consists of a film-like sheet sensor and a control unit.

\section{Seizure Monitor}

Motion Monitor is projected to detect faster, unnatural movements that may occur during the night, and will sound a high-frequency alert if these motions continue past a preset amount of time. The Emfit MM, Motion Monitor consists of a film-like sheet sensor and a control unit.

\section{RPI}

The Raspberry Pi is a bank card size microcontroller withthe features of a small PC and is extremely popular for development purposes because it offers the entire Linux server and peripheral device connectivity on a single chip and is very cost-effective.

\section{7. $\mathrm{MCP3008}$}

The MCP3008 is a low cost 8-channel 10-bit analogue to digital converter. The exactitude of this ADC is similar to that of an Arduino Uno, and with 8 channels you can read quite a few analog signals from the Pi.

\section{Level -1}

The algorithm [7] for monitoring of patient's health will be implemented as follows:- Firstly, in level-1, we will obtain the unprocessed data from several IoT devices and will be stacked away on the server. These devices include several sensors such as Vibration sensor, Temperature sensor and BP sensor. Because many of the sensors produce analog output that cannot be used by raspberry pi, we will firstly convert or change the analog values into digital form and then we will use the convertor IC. After this, Linux OS will be installed on the 
raspberry pi. The code in python will record the values of the sensors and modify them in the database at regular intervals.

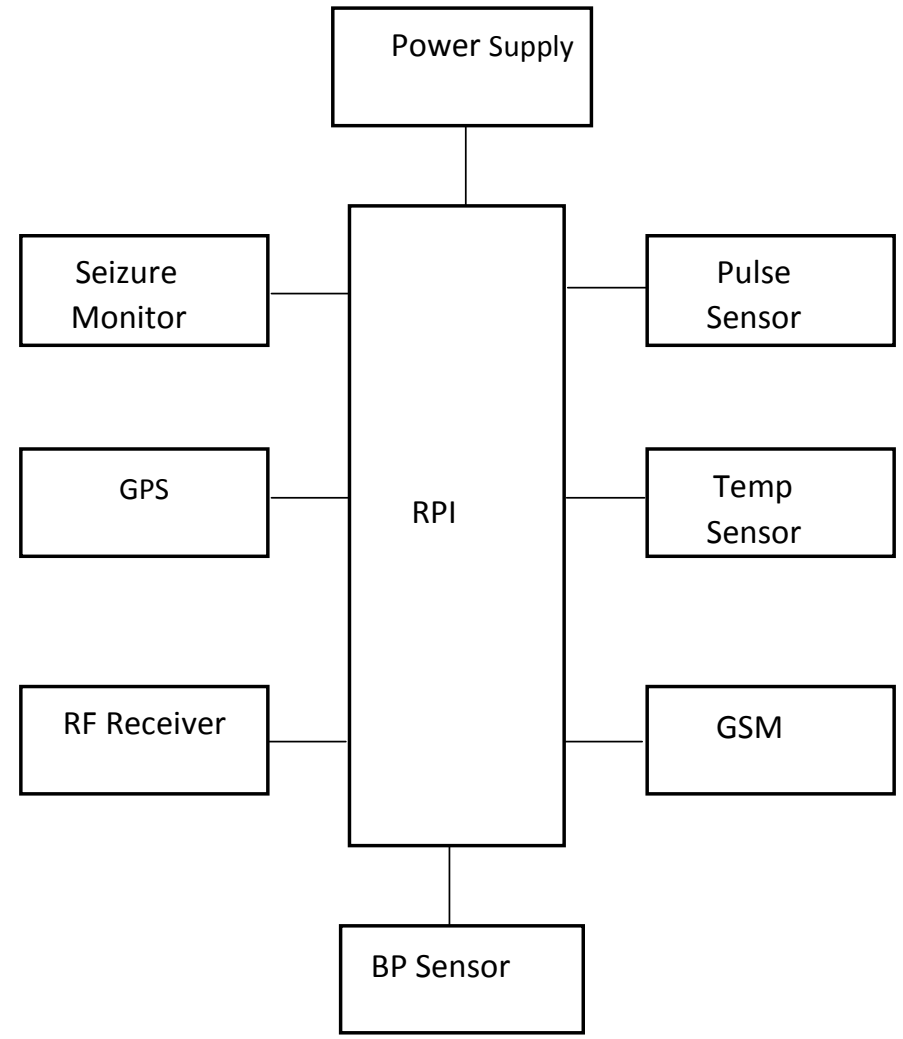

Fig. 4.1 Proposed System at the end of Patient

After this, we will obtain the relevant information as a result of the data stored by undergoing the following steps- filtering, classifying and then categorizing the data. This resulted information is nothing simply the patient's real-time health information and symptoms that will be appearing in the patient. It will be further used in the next level to predict if the patient is suffering from any kind of disease. This helps to make the system smart and efficient.

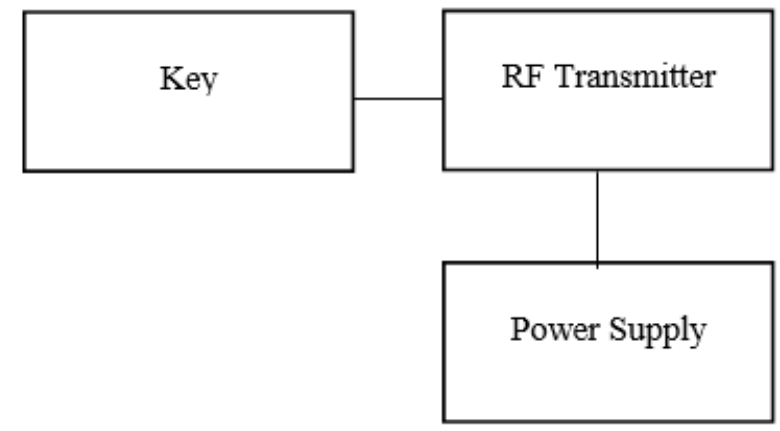

Fig 4.2 Proposed System at end of Monitor 


\section{RESULTS}

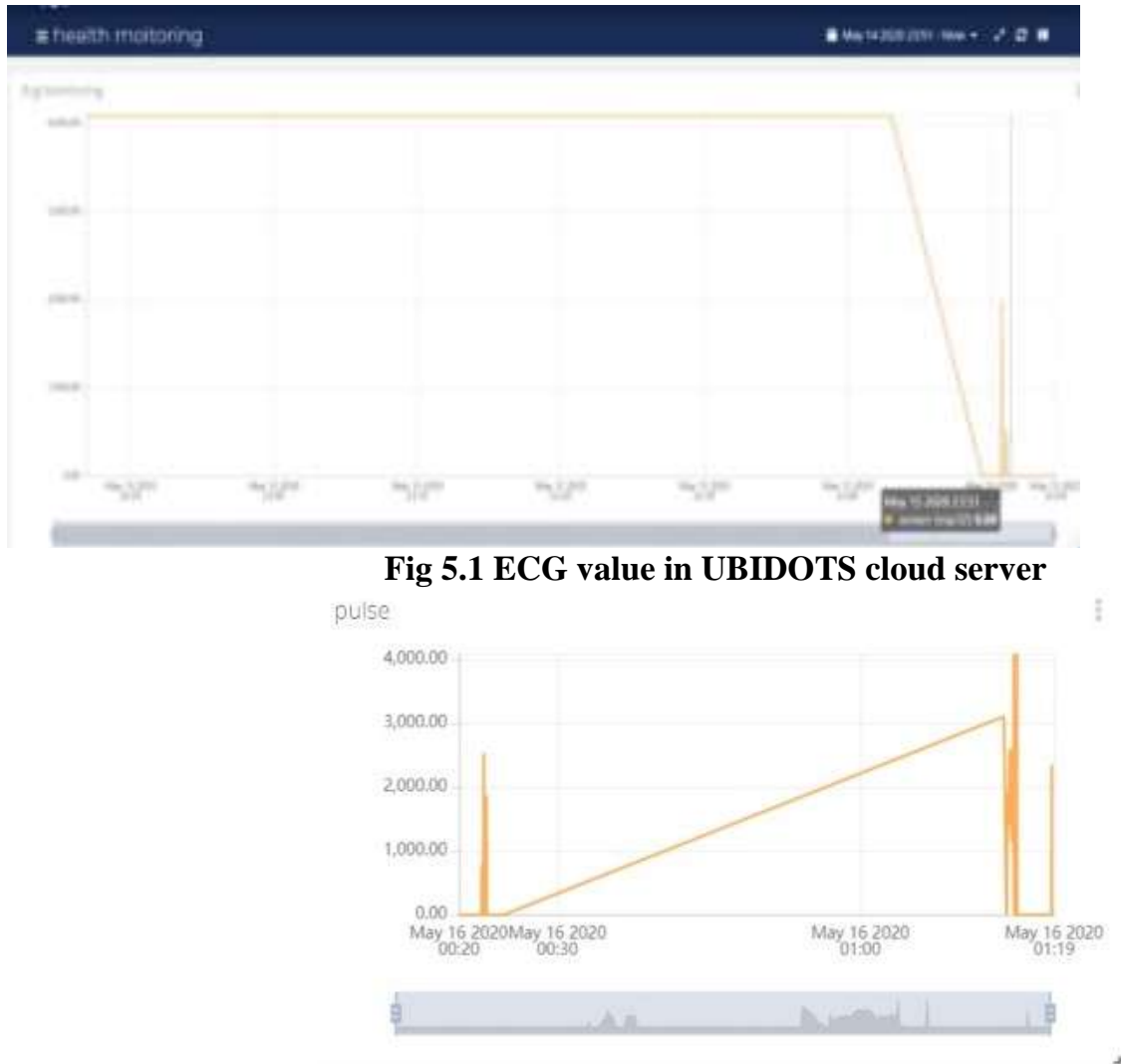

Fig 5.2 Pulse value in UBIDOTS cloud server

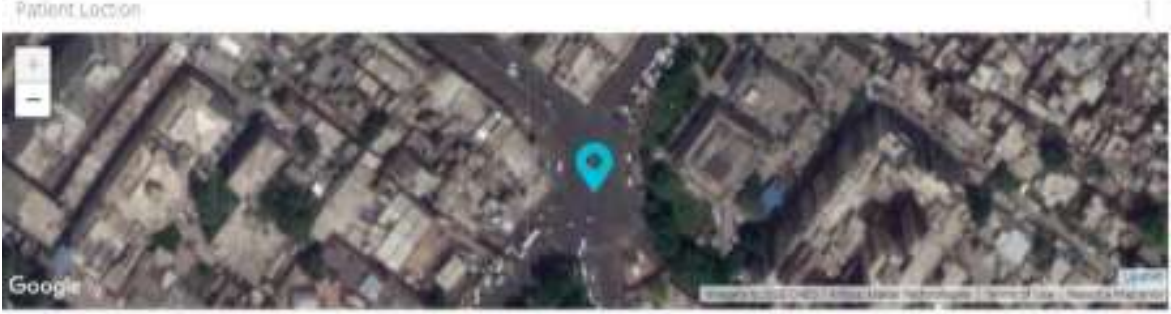

Fig 5.3 Patient's live location in UBIDOTS cloud server

\section{CONCLUSION}

In this paper, an automatic system is proposed that guarantees a constant monitoring of various health parameters and prediction of any kind of disease or disorder that prevents the patient from the pain of paying frequent visits to the hospitals. The proposed system can be set-up in the hospitals and massive amount of data can be obtained and stored in the online database. Even the consequences can be made to be accessed from mobile through an application.

The system can be further improved further by adding artificial intelligence system components to facilitate the doctors and the patients. The data, incorporating medical history of many patients' parameters and corresponding results, can be explored using data mining, in search of consistent patterns and systematic relationships in the disease. For instance, if a patient's health parameters are converting in the same form as those of a former patient in the database, the aftermaths can also be estimated. 


\section{VII.REFERENCES}

[1] N.V.Lopes,F.Pinto,P.Furtado,andJ.Silva, "Iot architecture proposal for handicapped people”, 2014 IEEE 10th International Conference on Wireless Mobile Ciphering, Networking Communications , pp. 152-158, Oct2014.

[2] B. G. Ahn, Y. H. Noh, and D. U. Jeong, "Smart chair based on multi heart rate detection system", 2015 IEEE SENSORS, pp. 1-4, Nov2015.

[3] S. H. Almotiri, M. A. Khan, and M. A. Alghamdi, "Mobile health (m-health) system in the contextof IoT", 2016 IEEE 4th International Conference on Future Internet of Things and Cloud Workshops (FiCloudW), pp. 39-42, Aug2016.

[4] T. S. Barger, D. E. Brown, and M. Alwan, "Healthstatus monitoring through analysis of behavioral patterns" IEEE Transactions on Systems, Man, and Cybernetics - Part A:Systems and Humans, pp.22-27, Jan2005.

[5] I. Chiuchisan, H. N. Costin, and O. Geman, "Adopting the internet of things technologies in health care systems", International Conference and Exposition on Electrical and Power Engineering (EPE), pp. 532- 535, Oct2014.

[6] A. Dwivedi, R. K. Bali, M. A. Belsis, R. N. G. Naguib,P. Every, and N. S. Nassar, "Towards a practical healthcare information security model for healthcare institutions", 4th International IEEE Conference on Applications in Biomedicine, pp. 114-117, April2003.

[7] S. Tyagi, A. Agarwal, and P. Maheshwari, "A conceptual framework for IoT-based healthcare system using cloud computing”, 2016 6th International Conference - Cloud System and Big Data Engineering , pp.503-507. 